\title{
Quantitative myocardial perfusion imaging using a step arterial-input function
}

\author{
Richard B Thompson ${ }^{1 *}$, Justin Grenier ${ }^{1}$, Emer Sonnex ${ }^{2}$, Richard Coulden ${ }^{2}$ \\ From 19th Annual SCMR Scientific Sessions \\ Los Angeles, CA, USA. 27-30 January 2016
}

\begin{abstract}
Background
Modern MRI myocardial perfusion protocols use rapid venous bolus injections, typically $3-5 \mathrm{ml} / \mathrm{s}$ of $5-15 \mathrm{ml}$ of agent over a few seconds. The resulting arterial input functions are rapidly varying with high agent concentrations (Fig. 1A and $1 \mathrm{~B}$ ) and thus typically require high temporal resolution acquisitions $(\sim 1 \mathrm{sec})$, custom pulse sequences and complex processing methods for perfusion quantification. A new myocardial perfusion approach, based on a pseudo step arterial-input function (Magn Reson Med. 2005 Aug;54(2):289-98), is introduced that offers simplified and lower concentration input functions, simplified quantitative data processing and reduced demands for high temporal resolution.
\end{abstract}

\section{Methods}

Numerical simulations of whole body vascular systems were used to design optimized venous injection protocols for the generation of step-input-like arterial-input functions targeting the idealized step-input function show in Fig. 1C. A two-compartment numerical model was used to estimate myocardial contrast agent concentration dynamics for conventional (bolus) and step-input protocols.

In-vivo experiments were performed on a Siemens Aera 1.5T (Siemens Healthcare, Erlangen, Germany). ECGgated saturation-recovery ( $\mathrm{TS}=100 \mathrm{~ms}$ ) bSFFP images were acquired for 120 heartbeats (1 image/beat, diastasis). Matrix size $224 \times 136$, rate 2 GRAPPA, $8 \mathrm{~mm}$ slice, 1.03 $\mathrm{ms} \mathrm{TE}, 2.5 \mathrm{~ms} \mathrm{TR}, 70^{\circ} \mathrm{flip}$. All contrast injections were single dose $(0.1 \mathrm{mmol} / \mathrm{kg})$ of Magnevist (Bayer). In-vivo data was acquired in 3 healthy controls and 3 CAD patients, all $\sim 90$ days post MI (LVEF $=45 \%-66 \%$, 61$92 \mathrm{~kg}$ ). Blood/tissue signal intensities were converted to contrast agent concentrations using a Bloch equation look-up-table approach and myocardial perfusion was estimated with an exponential deconvolution approach.

\section{Results}

Optimized venous injection protocols comprised decaying injection rates over $\sim 1 \mathrm{~min}$. with contrast agent dilution to $\sim 60 \mathrm{ml}$ (same protocol for all subjects). Sample blood and tissue time-intensity curves (normalized to baseline) in a healthy subject are shown in Fig. 1B and 1D, for a standard rapid bolus and an optimized step-input injection protocol. Fig. 2A shows arterial inputs for all subjects, and a sample perfusion map in a healthy control and patient are shown Fig. 2B and 2C.

\section{Conclusions}

A generalizable injection protocol can generate a pseudo arterial step-input function for a range of subject sizes and heart function, offering several advantages over conventional bolus injections: slower tissue dynamics enable multi-slice imaging with single-slice per heart-beat acquisitions, lower concentrations mitigate $\mathrm{T}_{2}{ }^{*}$ and $\mathrm{T}_{1}$ saturation effects and long injection duration avoids recirculation effects. The conventional short tissue "dynamic" window ( 10 seconds, Fig. 1B inset) reflects complex bolus injection dynamics; the pseudo-step arterial input reveals a longer window ( $\sim 60$ seconds, Fig. 1D) over which the contrast agent redistributes to the tissue via perfusion (as predicted with compartmental modeling in Fig. 1C).

\footnotetext{
Authors' details

${ }^{1}$ Biomedical Engineering, University of Alberta, Edmonton, AB, Canada. ${ }^{2}$ Radiology and Diagnostic Imaging, University of Alberta, Edmonton, AB, Canada.
} 


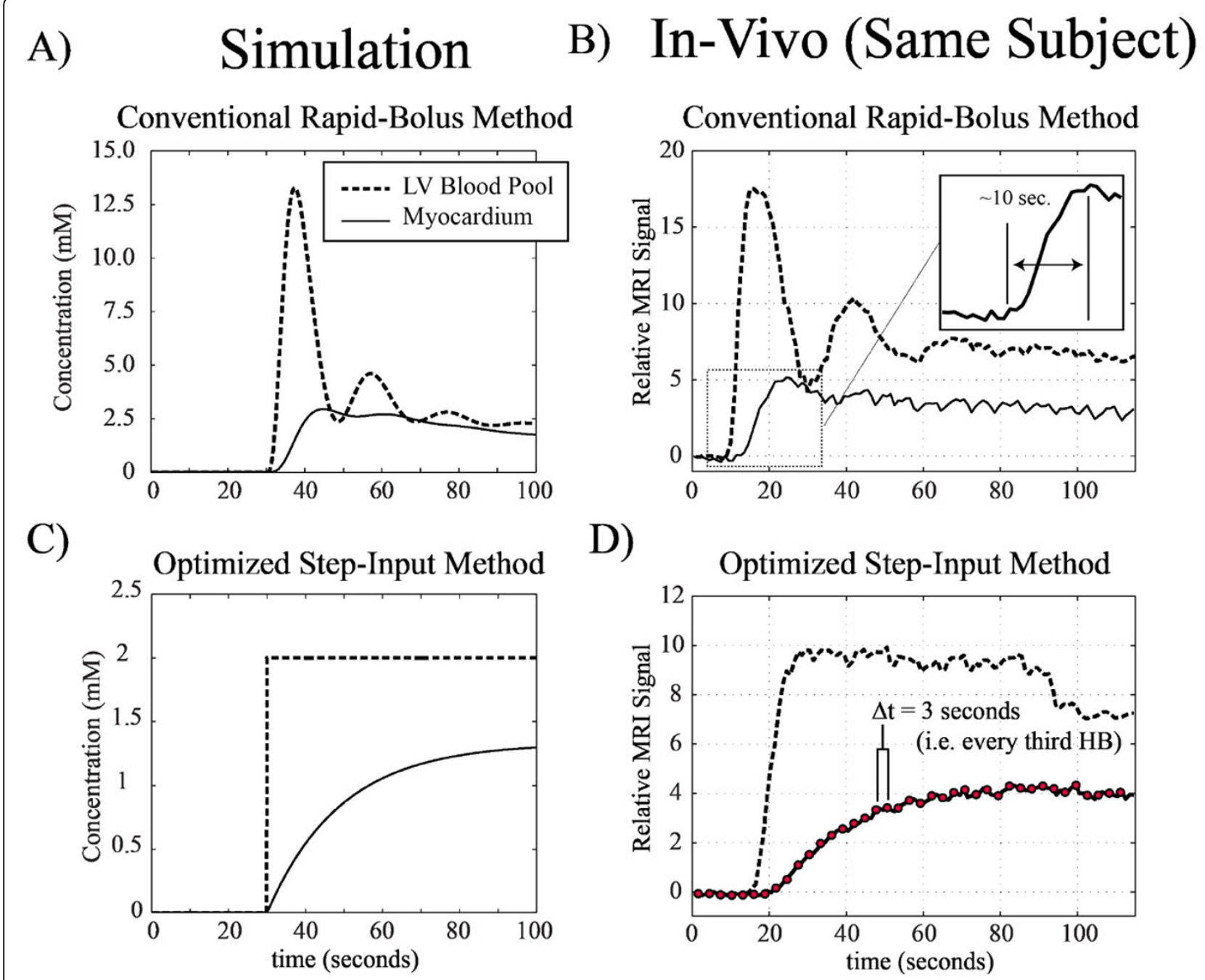

Figure 1 A) Simulated arterial input and tissue contrast agent concentration (based on $5 \mathrm{ml} / \mathrm{s}$ bolus injection and $1 \mathrm{ml} / \mathrm{g} / \mathrm{min}$ perfusion). B) In-vivo blood (LV pool) and myocardial signal (normalized to baseline) for a bolus injection (XX ml at $5 \mathrm{ml} / \mathrm{s}$ ) in a healthy control. C) Myocardial tissue response for an idealized step-input for $1 \mathrm{ml} / \mathrm{g} / \mathrm{min}$ perfusion. D) In-vivo blood (LV pool) and myocardial signal (normalized to baseline) for an optimized pseudo-step-input protocol (same subject as B).

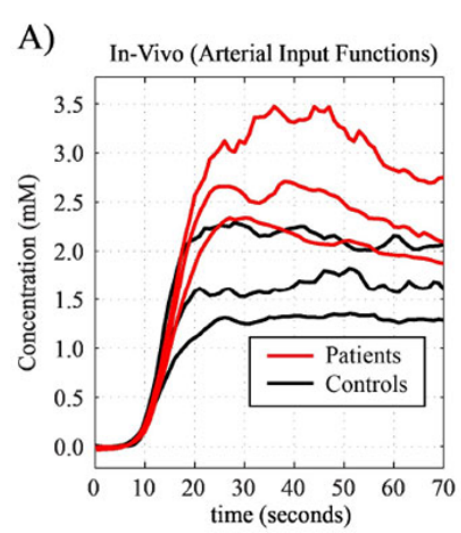

B) Perfusion (Healthy Control)

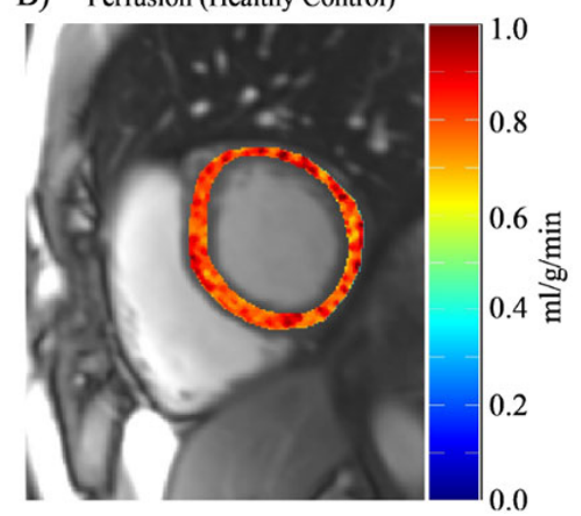

C) $\quad$ Perfusion $(\mathrm{CAD})$

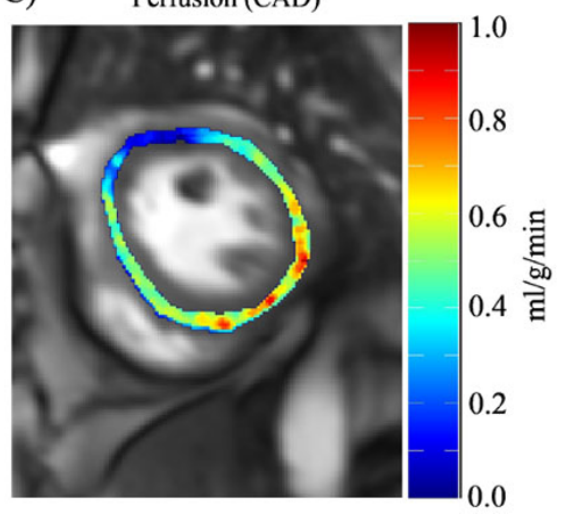

Figure $2 \mathrm{~A}$ ) Left ventricular arterial input functions for the 6 study subjects using the optimized step input venous injection protocol. Sample quantitative perfusion images for a healthy control subject and a patient with coronary artery disease (CAD) are shown in B) and C), respectively. 
doi:10.1186/1532-429X-18-S1-011

Cite this article as: Thompson et al: Quantitative myocardial perfusion imaging using a step arterial-input function. Journal of Cardiovascular Magnetic Resonance 2016 18(Suppl 1):011.

Submit your next manuscript to BioMed Central and take full advantage of:

- Convenient online submission

- Thorough peer review

- No space constraints or color figure charges

- Immediate publication on acceptance

- Inclusion in PubMed, CAS, Scopus and Google Scholar

- Research which is freely available for redistribution 